\title{
Pain sensitivity mediates the relationship between stress and headache intensity in chronic tension-type headache
}

\author{
Stuart Cathcart $\mathrm{PhD}^{1}$, Navjot Bhullar $\mathrm{PhD}^{2}$, Maarten Immink $\mathrm{PhD}^{3}$, Chris Della Vedova $\mathrm{PhD}^{4}$, John Hayball $\mathrm{PhD}^{4}$
}

\begin{abstract}
S Cathcart, N Bhullar, M Immink, C Della Vedova, J Hayball. Pain sensitivity mediates the relationship between stress and headache intensity in chronic tension-type headache. Pain Res Manage 2012;17(6):377-380.
\end{abstract}

BACKGROUND: A central model for chronic tension-type headache $(\mathrm{CTH})$ posits that stress contributes to headache, in part, by aggravating existing hyperalgesia in CTH sufferers. The prediction from this model that pain sensitivity mediates the relationship between stress and headache activity has not yet been examined.

OBJECTIVE: To determine whether pain sensitivity mediates the relationship between stress and prospective headache activity in CTH sufferers.

METHOD: Self-reported stress, pain sensitivity and prospective headache activity were measured in $53 \mathrm{CTH}$ sufferers recruited from the general population. Pain sensitivity was modelled as a mediator between stress and headache activity, and tested using a nonparametric bootstrap analysis.

RESULTS: Pain sensitivity significantly mediated the relationship between stress and headache intensity.

CONCLUSIONS: The results of the present study support the central model for CTH, which posits that stress contributes to headache, in part, by aggravating existing hyperalgesia in $\mathrm{CTH}$ sufferers. Implications for the mechanisms and treatment of $\mathrm{CTH}$ are discussed.

Key Words: Chronic tension-type headache; Mediation; Pain sensitivity; Stress

Stress is known to be a contributing factor to chronic tension-type Sheadache (CTH), with research indicating that mental stress is the most commonly reported trigger and aggravating factor of a CTH episode $(1,2)$. The mechanisms by which stress contributes to CTH are not clearly understood. Recent models of CTH pathology propose abnormalities in the central nervous system processing of pain in $\mathrm{CTH}$ sufferers, as indicated by increased sensitivity to mechanical, thermal and electrical noxious stimulation (1-13).

Because stress has been demonstrated to affect pain processing throughout the central nervous system $(1,2,9,10,13)$, one hypothesis for the mechanism by which stress contributes to CTH is that it aggravates already increased pain sensitivity in CTH sufferers $(1,2)$. Correlations among pain sensitivity, stress and headache activity (3-6), and evidence that both stress and pain sensitivity predict headache activity, support this central model $(7,8)$. Furthermore, experimentally inducing mental stress has been demonstrated to increase pain sensitivity and headache intensity in CTH sufferers $(9,10)$.

If stress aggravates headache by increasing pain sensitivity in $\mathrm{CTH}$ sufferers, then pain sensitivity should mediate the relationship between stress and subsequent headache. To date, no studies have tested this hypothesis. Such an examination is important for understanding the mechanisms leading to $\mathrm{CTH}$ and improving treatment for $\mathrm{CTH}$, given that stress is the most commonly reported aggravating factor for headache $(9,11,12)$, pain sensitivity is increased and considered to be a pathophysiological factor in CTH $(2,9,12,13)$ and behavioural treatments for CTH typically focus on stress management $(14,15)$. The present study, therefore, used mediation analyses to determine whether pain sensitivity mediates the relationship between stress and prospective

\section{La sensibilité à la douleur médiatrice entre le stress et l'intensité des céphalées de tension chroniques}

HISTORIQUE : D'après un modèle central de céphalées de tension chroniques (CTC), le stress contribue aux céphalées, en partie, en aggravant l'hyperalgie existante chez les personnes ayant ce type de céphalées. La prédiction de ce modèle, selon laquelle la sensibilité à la douleur est médiatrice entre le stress et l'activité céphalique, n'a pas encore été examinée. OBJECTIF : Déterminer si la sensibilité à la douleur est médiatrice entre le stress et l'activité céphalique prospective chez les personnes ayant des CTC.

MÉTHODOLOGIE : Les chercheurs ont mesuré le stress autodéclaré, la sensibilité à la douleur et l'activité céphalique prospective chez 53 personnes ayant des CTC recrutées au sein de la population générale. Ils ont modélisé la sensibilité à la douleur sous forme de médiateur entre le stress et l'activité céphalique, et ont mis ce modèle à l'essai au moyen d'une analyse d'amorce non paramétrique.

RÉSULTATS : La sensibilité à la douleur est médiatrice entre le stress et l'intensité de la céphalée de manière significative.

CONCLUSIONS : Les résultats de la présente étude appuient le modèle central de CTC, selon lequel le stress contribue aux céphalées, en partie, en aggravant l'hyperalgésie existante chez les personnes ayant des CTC. Les répercussions sur les mécanismes et le traitement des CTC sont exposées.

headache activity in CTH sufferers. It was hypothesized that the relationship between stress and prospective headache activity would be mediated by pain sensitivity.

\section{METHODS}

\section{Participants}

Participants were recruited from the general population through advertisements in local media requesting volunteers for a study on headaches. Of 94 volunteers, 56 met the inclusion criteria and were included in the study. Written consent was obtained from each subject and the study was approved by the Human Research Ethics Committee at the University of South Australia (Adelaide, Australia). Potential participants underwent a diagnostic interview based on the criteria outlined in the International Classification of Headache Disorders, Second Edition (16). Individuals satisfying the following criteria for CTH were included in the study:

- headache occurring on $\geq 15$ days per month on average for less than three months

- headache has at least two of the following characteristics: o bilateral location

$\circ$ pressing/tightening (nonpulsating) quality

$\circ$ mild or moderate intensity

- headache is not aggravated by routine physical activity

- headache lasts hours or may be continuous

- no more than one of photophobia, phonophobia or mild nausea

- neither moderate or severe nausea nor vomiting

- headache is not attributable to another disorder

${ }^{1}$ Centre for Applied Psychology, Faculty of Health, University of Canberra, Canberra; ${ }^{2}$ Centre for Health Initiatives, University of Wollongong,

Wollongong; ${ }^{3}$ School of Health Sciences; ${ }^{4}$ School of Pharmacy and Medical Sciences, University of South Australia, Adelaide, Australia

Correspondence: Dr Stuart Cathcart, Centre for Applied Psychology, University of Canberra, Bruce 2601, Australia.

Telephone 61-2-6202-3609, fax 61-2-6206-3962, e-mail stuart.cathcart@canberra.edu.au 


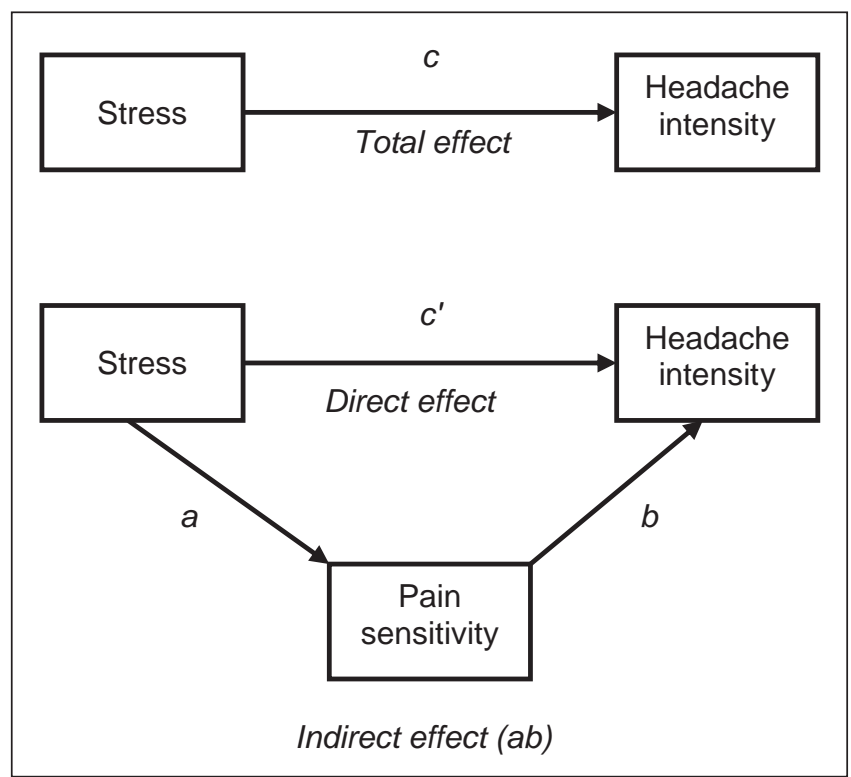

Figure 1) Mediation model for stress, pain sensitivity and headache. Path $c$ (total effect) is the total relationship between stress and headache. Path $c^{\prime}$ (direct effect) is the relationship between stress and headache controlling for pain sensitivity (ab). Path a is the relationship between stress and pain sensitivity. Path $b$ is the relationship between pain sensitivity and headache, adjusted for stress. Path $a b$ is the indirect relationship between stress and headache through pain sensitivity, which is algebraically equivalent to the difference between $c$ and $c^{\prime}(36)$

Additional inclusion criteria were that participants must have been between 18 and 65 years of age, not currently receiving (or having received in the past 12 months) intervention for headache, no psychiatric or major medical condition currently or in the past 12 months, no concurrent headache or pain symptoms or diagnoses other than CTH. Additionally, CTH subjects were required to not be taking or not have taken in the past three months any analgesic medication other than $\leq 1000 \mathrm{mg}$ per day of acetylsalicylic acid or paracetamol. None of the subjects reported taking prophylactic medication for headache or daily analgesic use. Data were complete for 53 of the original 56 participants. The sample had a mean ( \pm SD) age of $45.5 \pm 13.4$ years, and $34 \%$ of the participants were men.

\section{Measures}

Sociodemographic and clinical measures: Participants completed an in-house questionnaire assessing sociodemographic details and headache characteristics, and recorded headache activity for two weeks in a clinical diary detailed elsewhere (17). Measures calculated from the diary were mean headache intensity, mean headache duration (h) and mean headache frequency (number of days on which headache occurred).

Depression Anxiety Stress Scales-21: The Depression Anxiety Stress Scales-21 (DASS) is a 21-item self-report questionnaire containing subscales for measuring depression, anxiety and stress over the previous two weeks. Subjects respond to statements on a four-point scale indicating how much the statement applied to them over the previous two weeks. Sample items from the DASS Stress scale are 'I found it hard to wind down', 'I tended to over-react to situations', and 'I found it difficult to relax'. The DASS has high internal consistency and moderately high construct and convergent validity (18). Cronbach's alpha was high for all scales in the present study (stress $\alpha=0.92$, anxiety $\alpha=0.86$, depression $\alpha=0.90$ ).

Pain Sensitivity Questionnaire: The Pain Sensitivity Questionnaire (PSQ) (19) requires participants to respond to 17 items describing everyday painful situations by rating how painful they imagine such events would be on a scale ranging from 0 to 10 . The mean of the items is calculated to provide a total pain sensitivity score. Sample items from the PSQ are 'Imagine you burn your tongue on a very hot drink', 'Imagine you trap your finger in a drawer', 'Imagine you are wearing sandals and someone with heavy boots steps on your foot'. Adequate internal consistency and reliability has been demonstrated and validity has been confirmed against multiple quantitative sensory testing (QST) measures, including those used previously to assess pain sensitivity in headache (eg, threshold and suprathreshold response to experimental pressure, pinprick and thermal pain stimulation) (19). Cronbach's alpha for the present sample was high $(\alpha=0.94)$.

Headache diary: Participants were given a clinical diary detailed elsewhere (17) to record headache activity for two weeks postinterview. The diary recorded measures of mean headache intensity ( 0 to 5 scale), frequency (number of days on which headache occurred) and duration (h).

\section{Statistical analyses}

Statistical analyses were conducted using SPSS (IBM Corporation, USA) (20). Regression analysis to test for the mediation followed the procedures of Preacher and Hayes (21). First, correlations were conducted to test whether headache, stress and pain sensitivity were intercorrelated, as required for mediation analysis. To test the hypothesis that pain sensitivity would mediate the relationship between stress and headache intensity (Figure 1), a regression analysis was conducted using an SPSS macro developed by Preacher and Hayes (21). This macro conducts the same set of analyses recommended by Baron and Kenny (22) for assessing mediation, but also includes bootstrapped CIs for assessing the significance of indirect paths. Bootstrapped CIs are less biased than Sobel's test for examining the significance of indirect paths, particularly with sample sizes smaller than $n=80$ (21). A detailed description of bootstrapped tests of indirect effects can be found in Shrout and Bolger (23).

Mediation analysis assumes that the data represent the correct temporal sequence being modelled. In the present study, data were collected to allow temporal sequencing examining the hypothesis that pain sensitivity mediates the relationship between stress and prospective headache activity. Hence, during each subject's visit to the laboratory, the DASS was administered to measure stress over the previous two weeks, the PSQ was administered measuring current pain sensitivity, and subjects were given a headache diary to record headache activity over the following two weeks, which was then returned by mail to the researchers.

\section{RESULTS}

Stress, anxiety and depression levels measured using the DASS were in the normal range. From the headache diary, the mean headache intensity on a scale of 0 to 5 was $2.2 \pm 0.80$, the mean headache frequency (number of days over the two-week period on which a headache occurred) was $10.0 \pm 2.5$ days and the mean headache duration was $7.6 \pm 5.3 \mathrm{~h}$.

Table 1 presents the correlations among stress, pain sensitivity and headache activity. Higher scores on stress were related to greater pain sensitivity and headache intensity, and greater pain sensitivity was also related to greater headache intensity. Headache frequency and duration were not significantly correlated with either stress or pain sensitivity. Correlations between all relevant variables are a prerequisite for mediation analyses (22). Hence, mediation analyses were subsequently conducted with headache intensity as the only headache variable.

Path analysis revealed that stress was a significant predictor of pain sensitivity (path a in Figure 1: $\mathrm{B}=0.06, \mathrm{SE}=0.02, \mathrm{P}<0.01$ ) and headache intensity (path $c$ in Figure $1: B=0.05, S E=0.01, P<0.001$ ), and pain sensitivity was a significant predictor of headache intensity (path $\mathrm{b}$ in Figure 1: $\mathrm{B}=0.16, \mathrm{SE}=0.07, \mathrm{P}<0.05$ ). The direct effect of stress on headache (path $c^{\prime}$ in Figure 1) remained significant after controlling for pain sensitivity $(\mathrm{B}=0.04, \mathrm{SE} 0.01, \mathrm{P}<0.01)$ (Table 2). Overall, the model explained $32 \%$ of the variance in headache intensity (adjusted $\mathrm{R}^{2}=0.32, \mathrm{~F}[2,49]=12.72, \mathrm{P}<0.001$ ). The bootstrapped, biascorrected $95 \% \mathrm{CI}$ for the indirect effect of stress on headache through pain sensitivity (path ab in Figure 1) excluded zero (95\% CI 0.0012 to $0.0245)$, indicating that the effect was statistically significant. 
TABLE 1

Correlations among stress, pain sensitivity and headache activity in chronic tension-type headache sufferers

\begin{tabular}{lcccc}
\hline & Pain & \multicolumn{3}{c}{ Headache } \\
\cline { 3 - 5 } & sensitivity & Intensity & Frequency & Duration \\
\hline Stress & $0.34^{*}$ & $0.53^{\star \star}$ & 0.06 & 0.14 \\
Pain sensitivity & - & $0.42^{\star *}$ & 0.09 & 0.05 \\
Headache intensity & & - & 0.08 & 0.03 \\
Headache frequency & & & - & 0.14 \\
\hline
\end{tabular}

Stress was measured using the Depression Anxiety Stress Scales-21. Pain sensitivity was measured using the Pain Sensitivity Questionnaire. Mean headache intensity (0 to 5), frequency (number of days on which a headache occurred) and duration (h) were all recorded by participants in the 14-day headache diary. ${ }^{*} P<0.05$; ${ }^{*} P<0.01$

\section{DISCUSSION}

Stress is the most commonly reported trigger and aggravating factor of a primary headache episode $(1,9,11,12)$; however, the mechanisms underlying this relationship are not well understood. Increased pain sensitivity is a widely demonstrated feature of CTH and may be of pathophysiological significance, possibly reflecting sensitization in the myofascia, second-order spinal/trigeminal neurons, third-order thalamic or cortico-cortico projections, and/or increased pain cognitions $(2,6,9,12,13)$. Because stress is known to affect pain processing at all of these levels, a central model has been proposed, which posits that stress contributes to CTH by aggravating increased pain sensitivity in CTH sufferers $(1,2)$. Supporting this is evidence that both stress and pain sensitivity are related to headache activity in CTH sufferers (3-8) and experimentally-induced stress can increase pain sensitivity and induce headache $(9,10)$. No previous studies, however, have examined whether pain sensitivity mediates the relationship between stress and headache activity, as predicted by the central model. The present study demonstrated that self-reported pain sensitivity mediates the relationship between stress and prospective headache intensity in CTH sufferers. These results support the proposition that stress contributes to headache in CTH sufferers, in part, by aggravating pain sensitivity.

In the present study, pain sensitivity only partially mediated the relationship between stress and headache intensity. Thus, while a significant amount of variance (32\%) in the relationship between stress and headache intensity can be accounted for by pain sensitivity, the direct relationship between stress and headache remained significant (although significantly attenuated). This suggests that stress also contributes to headache intensity via other factors in addition to pain sensitivity. Alternatively, other aspects of pain sensitivity not assessed by the current measure could be involved. For example, muscle tenderness, which is increased in CTH and believed to primarily reflect peripheral sensitization, has previously been implicated in the relationship between stress and headache $(6,7,9)$. This suggests stress may also act on peripheral nociception to contribute to headache, a suggestion supported in recent animal models (24). In contrast, the PSQ is a measure of general pain sensitivity, which is increased in CTH $(2,6,12,13)$ and related to both stress and headache $(4,5,7,9)$.

To our knowledge, the present study was the first to use the PSQ in a chronic pain population. Pain sensitivity in chronic pain patients is typically assessed using quantitative sensory testing (QST) procedures including determination of detection, tolerance and suprathreshold responses to pressure, thermal or electrical stimulation. However, such procedures have several limitations. Correlations between measures may be low $(25,26)$, and the techniques require specialized equipment, trained staff and associated expense. Additionally, the procedures are aversive to patients. Although not previously used in CTH sufferers, the PSQ has been validated against multiple QST measures of pain sensitivity in healthy samples, including measures commonly used to demonstrate hyperalgesia in CTH such as pain detection and tolerance thresholds and suprathreshold pain ratings (19). The present findings indicate that the PSQ provides a simple, nonaversive method
TABLE 2

Path analyses among stress, pain sensitivity and headache intensity in chronic tension-type headache sufferers

\begin{tabular}{|c|c|c|c|c|}
\hline Model summary & Adj $R^{2}$ & $\mathrm{~F}$ & df & $\mathbf{P}$ \\
\hline & 0.32 & 12.72 & 2,49 & $<0.001$ \\
\hline Path analyses* & B & SE & $\mathrm{t}$ & $\mathbf{P}$ \\
\hline $\begin{array}{l}\text { Direct effect of stress on pain sensitivity } \\
\text { (path a) }\end{array}$ & 0.06 & 0.02 & 2.79 & $<0.01$ \\
\hline $\begin{array}{l}\text { Direct effect of pain sensitivity on } \\
\text { headache intensity (path b) }\end{array}$ & 0.16 & 0.07 & 2.12 & $<0.05$ \\
\hline $\begin{array}{l}\text { Total effect of stress on headache } \\
\text { intensity (path c) }\end{array}$ & 0.05 & 0.01 & 4.42 & $<0.001$ \\
\hline $\begin{array}{l}\text { Direct effect of stress on headache } \\
\text { intensity (path c') }\end{array}$ & 0.04 & 0.01 & 3.47 & $<0.01$ \\
\hline
\end{tabular}

*See Figure 1 for an illustration of the model. Stress was measured using the Depression Anxiety Stress Scales-21. Pain sensitivity was measured using the Pain Sensitivity Questionnaire. Mean headache intensity (0 to 5) was recorded by participants in the 14-day headache diary. Adj Adjusted

for examining pain sensitivity in CTH and, particularly, examining the role of pain sensitivity in stress and headache relationships.

The present methodology allows few conclusions to be drawn regarding the mechanisms involved in the mediating relationship of pain sensitivity in stress and headache. However, while stress could affect pain sensitivity throughout the pain system, it has previously been suggested that stress predominantly contributes to CTH via supraspinal mechanisms $(1,2,27)$. The present results offer some support for such a model. Particularly, most previous research demonstrating increased pain sensitivity in CTH has used QST measures. Using such methods, it is difficult to determine whether the increased pain response is due to sensitization in the myofascia, second-order spinal/trigeminal neurons, third-order thalamic or cortico-cortico projections, or increased pain cognitions. The PSQ provides a measure of pain sensitivity that does not rely on sensory stimulation. Hence, while lower-order (eg, peripheral) sensitization may contribute to increased pain report, the PSQ may be considered to predominantly reflect higher level (eg, cognitive) processing compared with QST, which reflects both sensory and cognitive processes. The present results are, therefore, consistent with the proposition that stress contributes to headache in CTH by aggravating predominantly supraspinal pain processes.

Uncertainty over biological mechanisms withstanding, the present results have important clinical implications. Because stress is the most commonly reported environmental correlate of headache, behavioural intervention for headache often focuses on stress reduction and management $(14,15)$. The present results indicate that the relationship between stress and headache intensity in CTH sufferers is mediated by pain sensitivity. Therefore, stress reduction per se may not be the primary mechanism of action in such interventions. Rather, the primary mechanism, at least for headache intensity, may be a reduction in pain sensitivity, or an alteration in the relationship between stress and pain sensitivity. Similarly, the present findings suggest that interventions for CTH may benefit from specifically focusing on altering the relationship between stress and pain sensitivity in CTH sufferers. For example, it has been demonstrated that headache sufferers have an attentional and memory bias toward stress and pain $(28,29)$ and enhanced associations between stress and pain (30). Speculatively, reducing such associations could alter the mediating effects of pain sensitivity in stress and headache relationships. Further research is needed to examine these possibilities.

\section{Limitations}

The present study had several limitations that need to be addressed in future research. It should be emphasized that although relationships among stress, pain sensitivity and prospective headache activity were examined, the mediation analyses were correlational, precluding any causal inference. An experimental design is needed to examine the causal significance of pain sensitivity in mediating stress and headache activity. 
Due to the relatively small sample size, we were unable to conduct a multiple group analysis based on sex. Some research suggests that stress and headache relationships and pain sensitivity differ according to sex $(4,5,11,12)$. Potentially, mediating effects of pain sensitivity in stress and headache relationships may also differ according to sex, and this needs to be explored in future research.

The use of single self-report measures for each of stress and pain sensitivity also present limitations, both with regard to identifying what particular aspects of stress and pain sensitivity are involved, and with regard to understanding the mechanisms underlying these relationships. However, self-report represents maximal integration of the stress and pain systems, and most previous research has used self-reported response to sensory stimulation to demonstrate increased pain sensitivity in CTH sufferers $(4-7,9,10)$, and hyperalgesic effects of stress on pain sensitivity in healthy humans $(31,32)$. Indeed, previous research has found effects of stress on pain report but not nociceptive reflex $(33,34)$. The present measures were, therefore, considered appropriate for this initial study, which requires replication and extension.

Finally, because the present study used a community-based sample, the present results may not be applicable to a clinical sample of CTH

\section{REFERENCES}

1. Cathcart S, Winefield AH, Lushington K, Rolan P. Stress and tension-type headache mechanisms. Cephalalgia 2010;30:1250-67.

2. Olesen J. Clinical and pathophysiological observations in migraine and tension-type headache explained by integration of vascular, supra-spinal and myofascial inputs. Pain 1991;46:125-32.

3. Materazzo F, Cathcart S, Pritchard D. Anger, depression and coping interactions in headache activity and adjustment: A controlled study. J Psychosom Res 2001;49:69-75

4. Ukestad LK, Wittrock DA. Pain perception and coping in female tension headache sufferers and headache-free controls. Health Psychol 1996;15:65-8.

5. Bishop KL, Holm JE, Borowiak DM, Wilson BA. Perception of pain in women with headache: A laboratory investigation of the influence of pain-related anxiety and fear. Headache 2001;41:494-9.

6. Mongini F, Ciccone G, Deregibus A, Ferrero L, Mongini T. Muscle tenderness in different headache types and its relation to anxiety and depression. Pain 2004;112:59-64.

7. Cathcart S, Pritchard D. Daily hassles and pain sensitivity in chronic tension-type headache sufferers. Stress Health 2008;24:123-7.

8. Bottos S, Dewey D. Perfectionists appraisal of daily hassles and chronic headache. Headache 2004;44:772-9.

9. Leistad RB, Sand T, Westgaard RH, Nilsen KB, Stovner LJ. Stress-induced pain and muscle activity in patients with migraine and tension-type headache. Cephalalgia 2006;26:64-73.

10. Cathcart S, Petkov J, Winefield AH, Lushington K, Rolan P. Central mechanisms of stress-induced headache. Cephalalgia 2010;30:285-95.

11. Nash JM, Thebarge RW. Understanding psychological stress, its biological processes, and impact on primary headache. Headache 2006;46:1377-86.

12. Fumal A, Schoenen J. Tension-type headache: Current research and clinical management. Lancet Neurology 2008;7:70-83.

13. Ashina S, Bendtsen L, Ashina M, Magerl W, Jensen R. Generalized hyperalgesia in patients with chronic tension-type headache. Cephalalgia 2006;26:940-8.

14. Martin PR, MacLeod C. Behavioral management of headache triggers: Avoidance of triggers is an inadequate strategy. Clin Psychol Rev 2009;29:483-95.

15. Kabat-Zinn J, Lipworth L, Burncy R, Sellers W. Four-year follow-up of a meditation based program for the self-regulation of chronic pain: Treatment outcomes and compliance. Clin J Pain 1986;2:159-73.

16. The International Classification of Headache Disorders, 2nd edn. Cephalalgia 2004;24(Suppl 1):9-160

17. Cathcart S, Pritchard D. Relationships between arousal related moods and episodic tension type headache: A biopsychological study. Headache 1998;38:214-21 sufferers. However, because most CTH sufferers do not seek treatment for their condition (12), the present results may be relevant to a large proportion of CTH sufferers. Further research is needed to assess the mediating role of pain sensitivity in stress and headache relationships in clinical samples.

\section{CONCLUSIONS}

The present study examined, for the first time, the mediating effects of pain sensitivity in the relationship between stress and headache. The results demonstrate that pain sensitivity mediates the relationship between stress and headache intensity in CTH sufferers. The results support a central model, which proposes that stress contributes to headache activity, in part, by aggravating existing hyperalgesia in CTH sufferers. Altering the relationship between stress and pain sensitivity may be a useful treatment focus for CTH.

ACKNOWLEDGEMENTS: The authors are grateful to Nicola Galatis and Margaret Mitchell for assistance with data collection. This research was funded in part by a Medical Advances Without Animals Trust project grant.

18. Lovibond SH, Lovibond PF. Manual for the Depression Anxiety Stress Scales, 2nd edn. Sydney: Psychology Foundation, 1995

19. Ruscheweyh R, Marziniak M, Stumpenhorst F, Reinholz J, Knecht S. Pain sensitivity can be assessed by self-rating: Development and validation of the Pain Sensitivity Questionnaire. Pain 2009;146:65-74

20. SPSS Inc. Statistical Package for the Social Sciences, Release 17.0, Vesta Services Inc, USA, 2009.

21. Preacher KJ, Hayes AF. Asymptotic and resampling strategies for assessing and comparing indirect effects in multiple mediator models. Behav Res Methods 2008;40:879-91.

22. Baron RM, Kenny DA. The moderator-mediator variable distinction in social psychological research: Conceptual, strategic, and statistical considerations. J Pers Soc Psychol 1986;51:1173-82.

23. Shrout PE, Bolger N. Mediation in experimental and nonexperimental studies: New procedures and recommendations. Psychol Methods 2002;7:422-45.

24. Khasar SG, Green PG, Levine J. Repeated sound stress enhances inflammatory pain in the rat. Pain 2005;116:79-86.

25. Bhalang K, Sigurdsson A, Slade GD, Maixner W. Associations among four modalities of experimental pain in women. J Pain 2005;6:604-11.

26. Hastie BA, Riley JL III, Robinson ME, et al. Cluster analysis of multiple experimental pain modalities. Pain 2005;116:227-37.

27. Cathcart S. A neural hypothesis for stress-induced headache. Med Hypotheses 2009;73:1011-13.

28. Liossi C, White P, Schoth DE. Time-course of attentional bias for threat-related cues in patients with chronic daily headache-tension type: Evidence for the role of anger. Eur J Pain 2011;15:92-8.

29. Kikuchi H, Yoshiuchi K, Miyasaka N, et al. Reliability of recalled self-report on headache intensity: Investigation using ecological momentary assessment technique. Cephalalgia 2006;26:1335-43.

30. Armstrong J, Wittrock D, Robinson M. Implicit associations in Tension-Type Headache: A cognitive analysis based on stress reactivity processes. Headache 2006;46:1281-90.

31. Zelman DC, Howland EW, Nichols SN, Cleeland CS. The effects of induced mood on laboratory pain. Pain 1991;46:105-11.

32. Caceres C, Burns JW. Cardiovascular reactivity to psychological stress may enhance subsequent pain sensitivity. Pain 1997;69:237-44.

33. French DJ, France CR, France JL, Arnott LF. The influence of acute anxiety on assessment of nociceptive flexion reflex threshold in healthy young adults. Pain 114;358-63.

34. Koh CW, Drummond PD. Dissociation between pain and the nociceptive blink reflex during psychological arousal. Clin Neurophys 2006;117;851-4. 


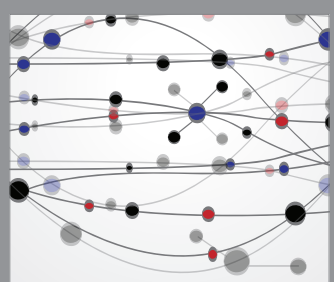

The Scientific World Journal
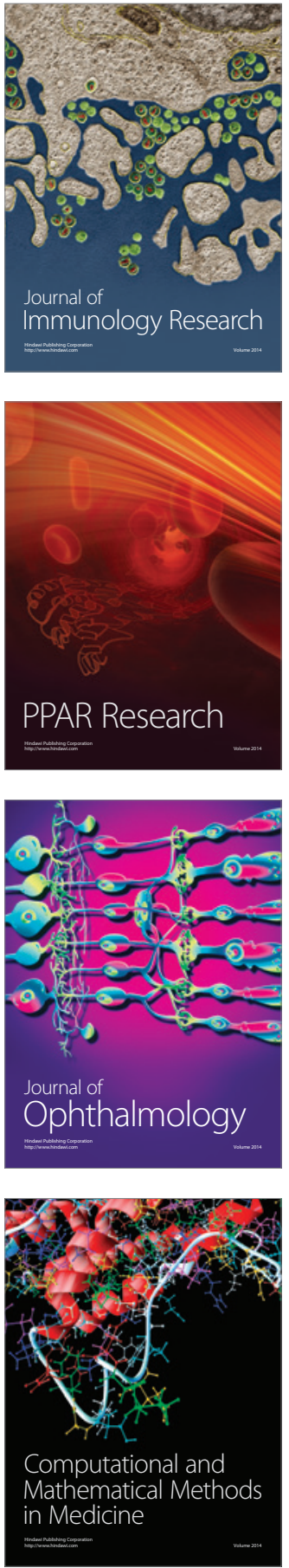

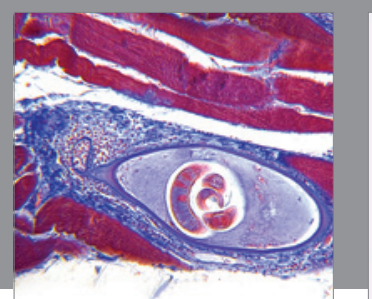

Gastroenterology Research and Practice

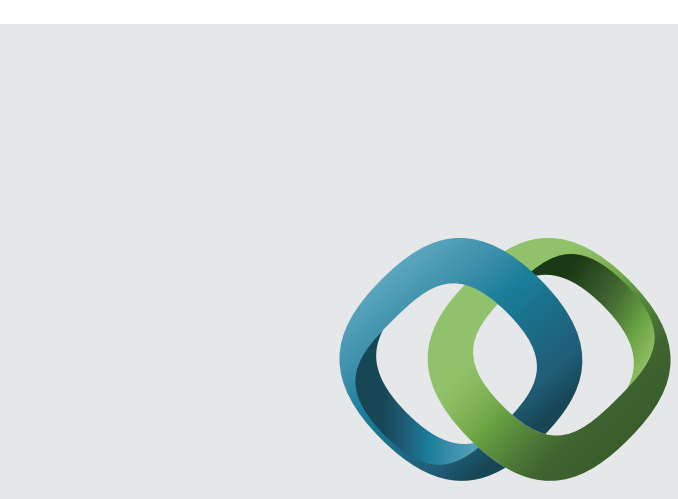

\section{Hindawi}

Submit your manuscripts at

http://www.hindawi.com
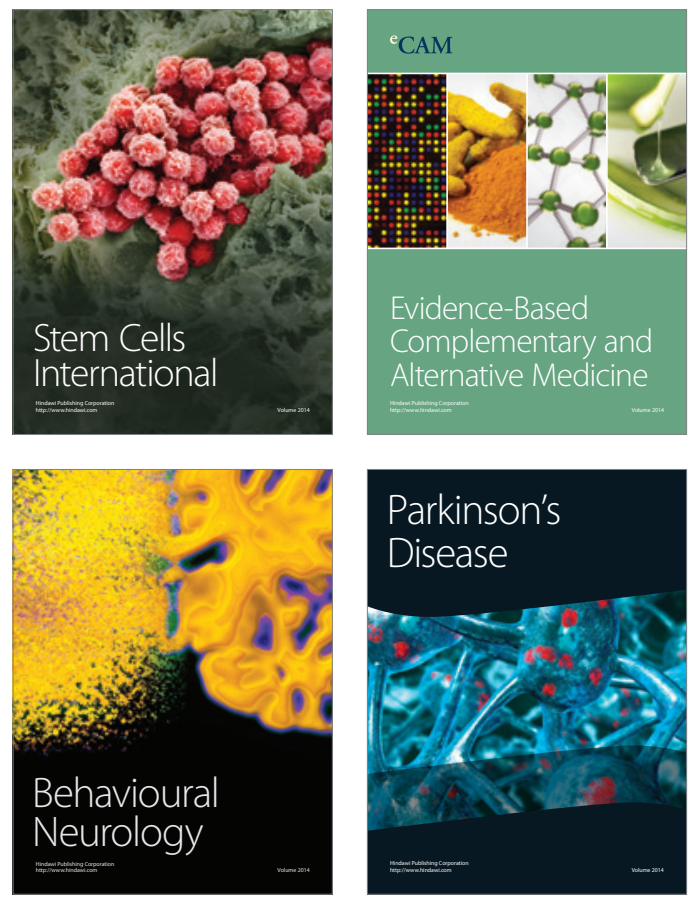
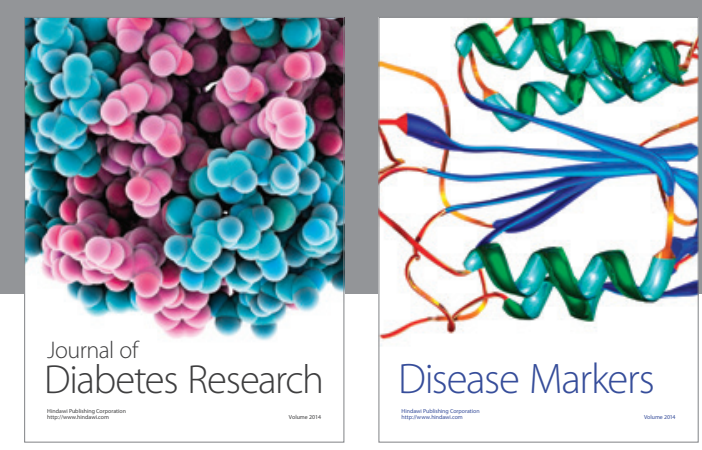

Disease Markers
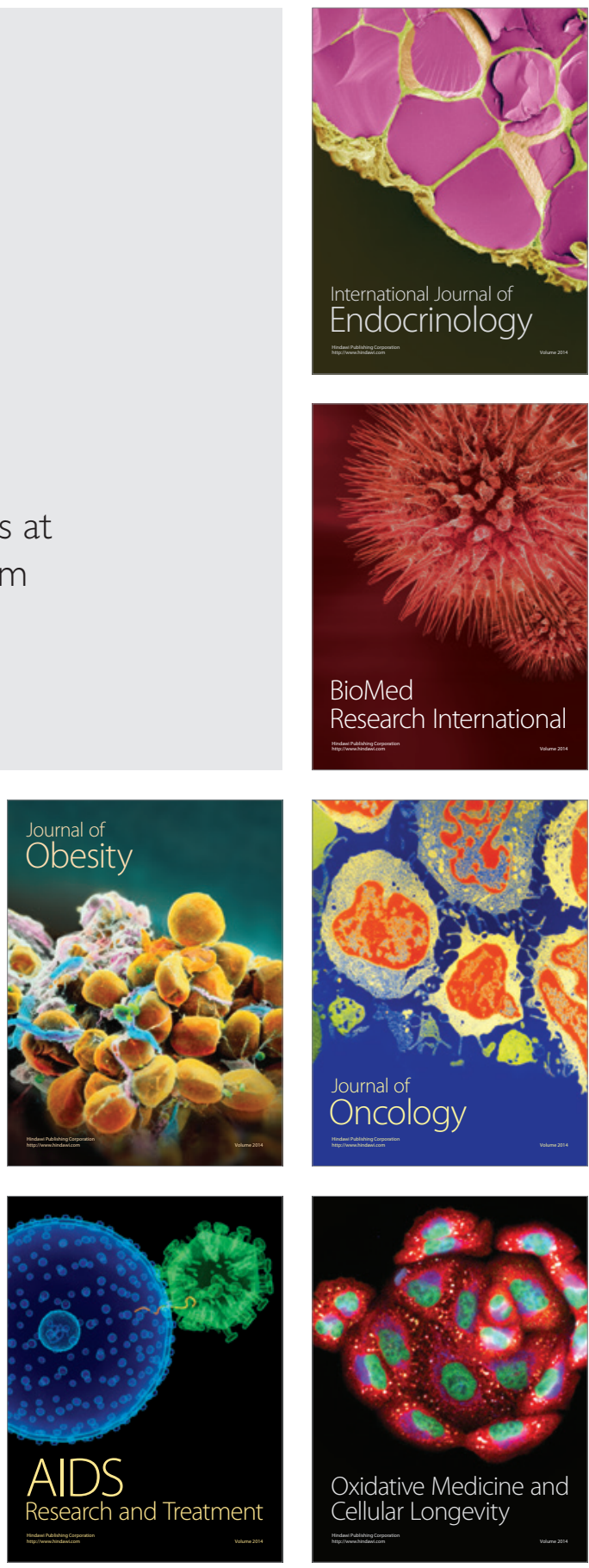